\title{
392.
}

\section{ON THE CONICS WHICH PASS THROUGH TWO GIVEN POINTS AND TOUCH TWO GIVEN LINES.}

[From the Quarterly Journal of Pure and Applied Mathematics, vol. vIII. (1867), pp. 211-219.]

LET $x=0, y=0$ be the equations of the given lines; $z=0$ the equation of the line joining the given points. We may, to fix the ideas, imagine the implicit constants so determined that $x+y+z=0$ shall be the equation of the line infinity.

Take $x-m y=0, x-n y=0$ as the equations of the lines which by their intersection with $z=0$ determine the given points. The equation of the conic is

$$
\{\sqrt{ }(m)+\sqrt{ }(n)\} \sqrt{ }(x y)=x+y \sqrt{ }(m n)+\gamma z,
$$

or, what is the same thing,

$$
(x-m y)(x-n y)+2\{x+y \sqrt{ }(m n)\} \gamma z+\gamma^{2} z^{2}=0,
$$

so that there are two distinct series of conics according as $\sqrt{ }(m n)$ is taken with the positive or the negative sign.

The equation of the chord of contact is

$$
x+y \sqrt{ }(m n)+\gamma z=0,
$$

which meets $z=0$ in the point $\{x+y \sqrt{ }(m n)=0, z=0\}$ that is in one of the centres of the involution formed by the lines $(x=0, y=0),(x-m y=0, x-n y=0)$. It is to be observed that the conic is only real when $m n$ is positive, that is (the lines and points being each real) the two points must be situate in the same region or in opposite regions of the four regions formed by the two lines: there are however other real cases; e.g. if the lines $x=0, y=0$ are real, but the quantities $m, n$ are conjugate imaginaries; included in this we have the circles which touch two real lines. 
To fix the ideas I take $m$ and $n$ each positive and $m n>1$; also $\mathrm{I}$ attend first to the series where $\sqrt{ }(m n)$ is taken positively. At the points where the conic meets infinity, we have

$$
\{\sqrt{ }(m)+\sqrt{ }(n)\} \sqrt{ }(x y)=x+y \sqrt{ }(m n)-\gamma(x+y),
$$

which gives two coincident points, that is the conic is a parabola, if

that is

$$
(1-\gamma)\{\sqrt{ }(m n)-\gamma\}=\frac{1}{4}\{\sqrt{ }(m)+\sqrt{ }(n)\}^{2},
$$

or

$$
\gamma^{2}-\gamma\{1+\sqrt{ }(m n)\}=\frac{1}{4}\{\sqrt{ }(m)-\sqrt{ }(n)\}^{2},
$$$$
\gamma=\frac{1}{2}[1+\sqrt{ }(m n) \pm \sqrt{ }\{(1+m)(1+n)\}],
$$

where it is to be noticed that

$$
\boldsymbol{\gamma}=\frac{1}{2}[1+\sqrt{ }(m n)+\sqrt{ }\{(1+m)(1+n)\}]
$$

is a positive quantity greater than $\sqrt{ }(m n)$, say $\gamma=p$,

$$
\boldsymbol{\gamma}=\frac{1}{2}[1+\sqrt{ }(m n)-\sqrt{ }\{(1+m)(1+n)\}]
$$

is a negative quantity, say $\gamma=-q, q$ being positive.

The order of the lines is as shown in fig. 1, see plate facing p. 52.

$$
\begin{aligned}
& \gamma=-\infty \text { to } \gamma=-q, \text { curve is ellipse; } \gamma=-q, \text { parabola } P_{2}, \\
& \gamma=-q \text { to } p, \text { curve is hyperbola; } \gamma=p, \text { parabola } P_{1}, \\
& \gamma=p \text { to } \gamma=\infty, \text { ellipse. }
\end{aligned}
$$

Resuming the equation

the coefficients are

$$
(x-m y)(x-n y)+2\{x+y \sqrt{ }(m n)\} \gamma z+\gamma^{2} z^{2}=0,
$$

$$
(a, b, c, f, g, h)=\left\{1, m n, \gamma^{2}, \gamma \sqrt{ }(m n), \gamma,-\frac{1}{2}(m+n)\right\},
$$

and thence the inverse coefficients are

$(A, B, C, F, G, H)=$

$$
\begin{aligned}
{\left[0,0,-\frac{1}{4}(m-n)^{2},\right.} & \left.-\frac{1}{2} \gamma\{\sqrt{ }(m)+\sqrt{ }(n)\}^{2},-\frac{1}{2} \gamma \sqrt{ }(m n)\{\sqrt{ }(m)+\sqrt{ }(n)\}^{2}, \frac{1}{2} \gamma^{2}\{\sqrt{ }(m)+\sqrt{ }(n)\}^{2}\right], \\
& K=-\frac{1}{4} \gamma^{2}\{\sqrt{ }(m)+\sqrt{ }(n)\}^{4},
\end{aligned}
$$

or, omitting a factor, the inverse coefficients are

$$
(A, B, C, F, G, H)=\left[0,0, \frac{1}{2 \gamma}\{\sqrt{ }(m)-\sqrt{ }(n)\}^{2}, 1, \sqrt{ }(m n),-\gamma\right] .
$$

Considering the line

$$
\lambda x+\mu y+\nu z=0,
$$

the coordinates of the pole of this line are

$$
\begin{aligned}
& \begin{array}{ll}
x: y: z= & -\gamma \mu+\sqrt{ }(m n) \nu
\end{array} \\
& :-\gamma \lambda+\nu \\
& : \sqrt{ }(m n) \lambda+\mu+\frac{1}{2 \gamma}\{\sqrt{ }(m)-\sqrt{ }(n)\}^{2} \nu,
\end{aligned}
$$


or (what is the same thing) introducing the arbitrary coefficient $k$, we have

$$
\begin{aligned}
& k x+\gamma \mu-\nu \sqrt{ }(m n)=0 \\
& k y+\gamma \lambda-\nu \quad=0 \\
& k z-\lambda \sqrt{ }(m n)-\mu-\frac{1}{2 \gamma}\{\sqrt{ }(m)-\sqrt{ }(n)\}^{2} \nu=0
\end{aligned}
$$

the first two equations give

that is

$$
k: \gamma:-1=\nu\{\mu-\lambda \sqrt{ }(m n)\}: \nu\{y \sqrt{ }(m n)-x\}: \lambda x-\mu y,
$$

$$
k=\frac{-\nu\{\mu-\lambda \sqrt{ }(m n)\}}{\lambda x-\mu y}, \quad \gamma=\frac{-\nu\{y \sqrt{ }(m n)-x\}}{\lambda x-\mu y},
$$

or, substituting this value of $\gamma$ in the third equation,

that is

$$
\frac{\nu\{\mu-\lambda \sqrt{ }(m n)\} z}{\lambda x-\mu y}+\{\mu+\lambda \sqrt{ }(m n)\}+\frac{\lambda x-\mu y}{x-y \sqrt{ }(m n)} \frac{\{\sqrt{ }(m)-\sqrt{ }(n)\}^{2}}{2}=0
$$

$$
\begin{aligned}
(\lambda x-\mu y)^{2} \cdot \frac{1}{2}\{\sqrt{ }(m)-\sqrt{ }(n)\}^{2}+\{x-y \sqrt{ }(m n)\}(\lambda x & -\mu y)\{\mu+\lambda \sqrt{ }(m n)\} \\
& +z\{x-y \sqrt{ }(m n)\} \nu\{\mu-\lambda \sqrt{ }(m n)\}=0,
\end{aligned}
$$

which is the equation of the curve, the locus of the pole of the line $\lambda x+\mu y+\nu z=0$ in regard to the conic

$$
(x-m y)(x-n y)+2\{x+y \sqrt{ }(m n)\} \gamma z+\gamma^{2} z^{2}=0 .
$$

In particular, if $\lambda=\mu=\nu=1$, then for the coordinates of the centre of the conic, we have

$$
x: y: z=-\gamma+\sqrt{ }(m n):-\gamma+1: \sqrt{ }(m n)+1+\frac{1}{2 \gamma}\{\sqrt{ }(m)-\sqrt{ }(n)\}^{2} ;
$$

and for the locus of the centre,

$(x-y)^{2} \cdot \frac{1}{2}\{\sqrt{ }(m)-\sqrt{ }(n)\}^{2}+(x-y)\{x-y \sqrt{ }(m n)\}\{1+\sqrt{ }(m n)\}+z\{x-y \sqrt{ }(m n)\}\{1-\sqrt{ }(m n)\}=0$,

so that the locus is a conic, and it is obvious that this conic is a hyperbola. Putting for greater simplicity

$$
\begin{aligned}
& x-y=X, \\
& x-y \sqrt{ }(m n)=Y, \\
& z \quad=Z,
\end{aligned}
$$

the equation of the curve of centres is

$$
X^{2} \cdot \frac{1}{2}\{\sqrt{ }(m)-\sqrt{ }(n)\}^{2}+X Y\{1+\sqrt{ }(m n)\}+Y Z\{1-\sqrt{ }(m n)\}=0,
$$

or, writing this under the form

$$
Y[X\{1+\sqrt{ }(m n)\}+\boldsymbol{Z}\{1-\sqrt{ }(m n)\}]+\frac{1}{2}\{\sqrt{ }(m)-\sqrt{ }(n)\}^{2} X^{2}=0,
$$


the equation is

where

$$
Y Q+X^{2}=0
$$

$$
\begin{aligned}
& X=x-y, \\
& Y=x-y \sqrt{ }(m n), \\
& Q=\frac{2}{\{\sqrt{ }(m)-\sqrt{ }(n)\}^{2}}[\{1+\sqrt{ }(m n)\}(x-y)+\{1-\sqrt{ }(m n)\} z]:
\end{aligned}
$$

these values give

$$
\begin{aligned}
x-y & =X, \\
x-y \sqrt{ }(m n) & =Y, \\
\{1-\sqrt{ }(m n)\} z & =\{\sqrt{ }(m)-\sqrt{ }(n)\}^{2} Q+2\{1+\sqrt{ }(m n)\} X,
\end{aligned}
$$

or, what is the same thing,

$$
\begin{aligned}
& \{1-\sqrt{ }(m n)\} x=\quad-\sqrt{ }(m n) X+Y, \\
& \{1-\sqrt{ }(m n)\} y= \\
& \{1-\sqrt{ }(m n)\} z=2\{1+\sqrt{ }(m n)\} X+Y \quad+\{\sqrt{ }(m)-\sqrt{ }(n)\}^{2} Q
\end{aligned}
$$

whence also

$$
\{1-\sqrt{ }(m n)\}(x+y+z)=\{1+\sqrt{ }(m n)\} X+2 Y+\{\sqrt{ }(m)-\sqrt{ }(n)\}^{2} Q,
$$

or the equation of the line infinity is

$$
\{1+\sqrt{ }(m n)\} X+2 Y+\{\sqrt{ }(m)-\sqrt{ }(n)\}^{2} Q=0,
$$

a formula which may be applied to finding the asymptotes and thence the centre of the conic

$$
Y Q+X^{2}=\overline{0}
$$

In fact we have identically

$$
\{2 k x+2 k y-(2 k+1) z\}^{2}-(1+4 k)(2 k x-z)^{2}=4 k^{2}(x+y+z)^{2}-4 k(1+4 k)\left(k x^{2}+y z\right),
$$

that is

$$
-4 k(1+4 k)\left(k x^{2}+y z\right)=\{2 k x+2 k y-(2 k+1) z\}^{2}-(1+4 k)(2 k x-z)^{2}-4 k^{2}(x+y+z)^{2},
$$

which, if $x+y+z=0$ is the equation of the line infinity, puts in evidence the asymptotes of the conic $k x^{2}+y z=0$. Hence writing $\alpha x, \beta y, \gamma z$ in the place of $x, y, z$ respectively, and $\frac{k \alpha^{2}}{\beta \gamma}=k^{\prime}$, that is, $k=\frac{\beta \gamma}{\alpha^{2}} k^{\prime}$, we have

$$
\begin{aligned}
-\frac{4 \beta \gamma}{\alpha^{2}} k^{\prime}\left(1+\frac{4 \beta \gamma}{\alpha^{2}} k^{\prime}\right) \beta \gamma\left(k^{\prime} x^{2}+y z\right) & =\left\{\frac{2 \beta \gamma}{\alpha} k^{\prime} x+\frac{2 \beta^{2} \gamma}{\alpha^{2}} k^{\prime} y-\left(2 \frac{\beta \gamma}{\alpha^{2}} k^{\prime}+1\right) \gamma z\right\}^{2} \\
& -\left(1+\frac{4 \beta \gamma}{\alpha^{2}} k^{\prime}\right)\left(\frac{2 \beta \gamma}{\alpha} k^{\prime} x-\gamma z\right)^{2}-4 \frac{\beta^{2} \gamma^{2}}{\alpha^{4}} k^{\prime 2}(\alpha x+\beta y+\gamma z)^{2}
\end{aligned}
$$


that is

$$
\begin{aligned}
&-4 \beta^{2} \gamma^{2} k^{\prime}\left(\alpha^{2}+4 \beta \gamma k^{\prime}\right)\left(k^{\prime} x^{2}+y z\right)=\left\{2 \alpha \beta \gamma k^{\prime} x+2 \beta^{2} \gamma k^{\prime} y-\left(2 \beta \gamma k^{\prime}+\alpha^{2}\right) \gamma z\right\}^{2} \\
&-\left(\alpha^{2}+4 \beta \gamma k^{\prime}\right)\left(2 \beta \gamma k^{\prime} x-\alpha \gamma z\right)^{2}-4 \beta^{2} \gamma^{2} k^{2}(\alpha x+\beta y+\gamma z)^{2},
\end{aligned}
$$

or, what is the same thing,

$-4 \beta^{2} k^{\prime}\left(\alpha^{2}+4 \beta \gamma k^{\prime}\right)\left(k^{\prime} x^{2}+y z\right)=\left\{2 \alpha \beta k^{\prime} x+2 \beta^{2} k^{\prime} y-\left(2 \beta \gamma k^{\prime}+\alpha^{2}\right) z\right\}^{2}$

$$
-\left(\alpha^{2}+4 \beta \gamma k^{\prime}\right)(2 \beta k x-\alpha z)^{2}-4 \beta^{2} k^{\prime 2}(\alpha x+\beta y+\gamma z)^{2},
$$

which, when $a x+\beta y+\gamma z=0$ is the equation of the line infinity, puts in evidence the asymptotes of the conic $k^{\prime} x^{2}+y z=0$.

Now writing $X, Y, Q$ in the place of $x, y, z ; k^{\prime}=1$, and $\alpha=\{1+\sqrt{ }(m n)\}, \beta=2$, $\boldsymbol{\gamma}=\{\sqrt{ }(m)-\sqrt{ }(n)\}^{2}$, we have

$$
\begin{aligned}
-16[\{1+ & \left.\sqrt{ }(\min )\}^{2}+8\{\sqrt{ }(m)-\sqrt{ }(n)\}^{2}\right]\left(Y Q+X^{2}\right) \\
= & {\left[4\{1+\sqrt{ }(m n)\} X+8 Y-\left(4\{\sqrt{ }(m)-\sqrt{ }(n)\}^{2}+\{1+\sqrt{ }(m n)\}^{2}\right) Q\right]^{2} } \\
& -\left[\{1+\sqrt{ }(m n)\}^{2}+8\{\sqrt{ }(m)-\sqrt{ }(n)\}^{2}\right][4 X-\{1+\sqrt{ }(m n)\} Q]^{2} \\
& -16\left[\{1+\sqrt{ }(m n)\} X+2 Y+\{\sqrt{ }(m)-\sqrt{ }(n)\}^{2} Q\right]^{2},
\end{aligned}
$$

and the asymptotes are

$$
\begin{aligned}
4\{1+\sqrt{ }(m n)\} X+ & 8 Y-\left[4\{\sqrt{ }(m)-\sqrt{ }(n)\}^{2}+\{1+\sqrt{ }(m n)\}^{2}\right] Q \\
& = \pm \sqrt{\{1+\sqrt{ }(m n)\}^{2}+8\{\sqrt{ }(m)-\sqrt{ }(n)\}^{2}}[4 X-\{1+\sqrt{ }(m n)\} Q]
\end{aligned}
$$

At the centre

$$
\begin{aligned}
& 4\{1+\sqrt{ }(m n)\} X+8 Y-\left[4\{\sqrt{ }(m)-\sqrt{ }(n)\}^{2}+\{1+\sqrt{ }(m n)\}^{2}\right] Q=0 \\
& 4 X-\{1+\sqrt{ }(m n)\} Q=0
\end{aligned}
$$

but the first equation is

$$
\{1+\sqrt{ }(m n)\}[4 X-Q\{1+\sqrt{ }(m n)\}]+8 Y-4\{\sqrt{ }(m)-\sqrt{ }(n)\}^{2} Q=0,
$$

so that we have

the first of these is

$$
4 X=\{1+\sqrt{ }(m n)\} Q, \quad 2 Y=\{\sqrt{ }(m)-\sqrt{ }(n)\}^{2} Q,
$$

$$
2\{\sqrt{ }(m)-\sqrt{ }(n)\}^{2}(x-y)-\{1+\sqrt{ }(m n)\}^{2}(x-y)-(1-m n) z=0,
$$

and the two together give

$$
2 X\{\sqrt{ }(m)-\sqrt{ }(n)\}^{2}-\{\mathbf{1}+\sqrt{ }(m n)\} Y=0,
$$

so that we have

$$
\begin{aligned}
& 2\{\sqrt{ }(m)-\sqrt{ }(n)\}^{2}(x-y)-\{1+\sqrt{ }(m n)\}\{x-y \sqrt{ }(m n)\}=0, \\
& {\left[\{1+\sqrt{ }(m n)\}^{2}-2\{\sqrt{ }(m)-\sqrt{ }(n)\}^{2}\right](x-y)+(1-m n) z=0,}
\end{aligned}
$$

to determine the coordinates of the centre. 
The equation of the chord of contact is

$$
x+y \sqrt{ }(m n)+\gamma z=0,
$$

which for $\gamma=1$ is parallel to $y=0$ and for $\gamma=\sqrt{ }(m n)$ is parallel to $x=0$. But the coordinates of the centre are

$$
x: y: z=-\gamma+\sqrt{ }(m n):-\gamma+1: \sqrt{ }(m n)+1+\frac{1}{2 \gamma}\{\sqrt{ }(m)-\sqrt{ }(n)\}^{2},
$$

which for $\gamma=1$ give

$$
y=0, x: z=-1+\sqrt{ }(m n): \sqrt{ }(m n)+1+\frac{1}{2}\{\sqrt{ }(m)-\sqrt{ }(n)\}^{2},=-2+2 \sqrt{ }(m n): 2+m+n,
$$

and for $\gamma=\sqrt{ }(m n)$ give

$$
x=0 \text {, }
$$

$y: z=1-\sqrt{ }(m n): \sqrt{ }(m n)+1+\frac{1}{2 \sqrt{ }(m n)}\{\sqrt{ }(m)-\sqrt{ }(n)\}^{2},=2-2 \sqrt{ }(m n): 2 \sqrt{ }(m n)+\frac{m+n}{\sqrt{ }(m n)}$.

The line drawn from the fixed point on the chord of contact to the centre has for its equation

$$
x+y \sqrt{ }(m n)+\gamma^{\prime} z=0,
$$

where, writing for $x, y, z$ the coordinates of the centre, we have

$$
-\gamma\{1+\sqrt{ }(m n)\}+2 \sqrt{ }(m n)+\gamma^{\prime}\left[\sqrt{ }(m n)+1+\frac{1}{2 \gamma}\{\sqrt{ }(m)-\sqrt{ }(n)\}^{2}\right]=0,
$$

that is

$$
\gamma^{\prime}=\frac{\gamma\{1+\sqrt{ }(m n)\}-2 \sqrt{ }(m n)}{1+\sqrt{ }(m n)+\frac{1}{2 \gamma}\{\sqrt{ }(m)-\sqrt{ }(n)\}^{2}},
$$

or, what is the same thing,

$$
\gamma^{\prime}-\gamma=\frac{-\gamma\{\sqrt{ }(m)+\sqrt{ }(n)\}^{2}}{\{\sqrt{ }(m)-\sqrt{ }(n)\}^{2}+2 \gamma\{1+\sqrt{ }(m n)\}},
$$

and consequently $\gamma^{\prime}=\gamma$ only for $\gamma=0$.

It is now easy to trace the corresponding positions of the chord of contact through the fixed point $\{x+y \sqrt{ }(m n)=0, z=0\}$, and of the centre on the hyperbola which is the curve of centres: see fig. 2 in the plate facing p. 52 .

The lines $O P_{2}, O L, O \Theta, O P_{1}, O X, O G, O H$ are positions of the chord of contact, and the points $P_{2}, L, \Theta, P_{1}, X, G, H$, on the hyperbola which is the curve of centres are the corresponding positions of the centre. 
Chord of Contact.

$O P_{2}$.

$O L(z=0)$.

$O \Theta$.

$O P_{1}$.

$O X\{x+y \sqrt{ }(m n)=0\}$.

$O G$ (parallel to $y=0$ ).

$O H$ (parallel to $x=0$ ) and so back to $O P_{2}$.

I have treated separately the case $\sqrt{ }(m n)=1$.

Consider the conics which touch the lines $y-x=0, y+x=0$ and pass through the points

$$
\left\{x=1, y=\sqrt{ }\left(1-c^{2}\right)\right\},\left\{x=1, y=-\sqrt{ }\left(1-c^{2}\right)\right\} .
$$

The equation is of the form

$$
y^{2}-x^{2}+k(x-\alpha)^{2}=0,
$$

and to determine $k$, we have

$$
1-c^{2}-1+k(1-\alpha)^{2}=0, \text { and therefore } k=\frac{c^{2}}{(1-\alpha)^{2}} .
$$

The equation thus becomes

that is

$$
(1-\alpha)^{2}\left(y^{2}-x^{2}\right)+c^{2}(x-\alpha)^{2}=0,
$$

$$
(1-\alpha)^{2} y^{2}+\left\{c^{2}-(1-\alpha)^{2}\right\} x^{2}-2 c^{2} \alpha x+c^{2} \alpha^{2}=0,
$$

or as this may be written

$$
(1-\alpha)^{2} y^{2}+\left\{c^{2}-(1-\alpha)^{2}\right\}\left\{x-\frac{c^{2} \alpha}{c^{2}-(1-\alpha)^{2}}\right\}^{2}-\frac{c^{2} \alpha^{2}(1-\alpha)^{2}}{c^{2}-(1-\alpha)^{2}}=0 .
$$

Hence the nature of the conic depends on the sign of $c^{2}-(1-\alpha)^{2}$, viz. if this be positive, or $\alpha$ between the limits $1+c, 1-c$, the curve is an ellipse,

which is positive,

$$
\begin{array}{ll}
x \text {-coordinate of centre } & =\frac{c^{2} \alpha}{c^{2}-(1-\alpha)^{2}}, \\
x \text {-semi-axis } & =\frac{ \pm c \alpha(1-\alpha)}{c^{2}-(1-\alpha)^{2}}, \\
y \text {-semi-axis } & =\frac{c \alpha}{\sqrt{\left\{c^{2}-(1-\alpha)^{2}\right\}}} .
\end{array}
$$

The coordinate of centre for $\alpha=1+c$ is $=+\infty$ (the curve being in this case a parabola $P_{1}$ ) and for $\alpha=1-c$ it is also $=+\infty$ (the curve being in this case a parabola $P_{2}$ ). The coordinate has a minimum value corresponding to $\alpha=\sqrt{ }\left(1-c^{2}\right)$, viz. this is $=\frac{1}{2}\left\{1+\sqrt{ }\left(1-c^{2}\right)\right\}$.

c. VI. 
Hence as $(\alpha)$ passes from $1+c$ to $\sqrt{ }\left(1-c^{2}\right)$, the coordinate of the centre passes from $\infty$ to its minimum value $\frac{1}{2}\left\{1+\sqrt{ }\left(1-c^{2}\right)\right\}$; in the passage we have $\alpha=1$ giving the coordinate $=1$, the conic being in this case a pair of coincident lines $(x-1)^{2}=0$. And as ( $x$ ) passes from the foregoing value $\sqrt{ }\left(1-c^{2}\right)$ to $1-c$, the coordinate of the centre passes from the 'minimum value $\frac{1}{2}\left\{1+\sqrt{ }\left(1-c^{2}\right)\right\}$ to $\infty$.

The curve is a hyperbola if $\alpha$ lies without the limits $1+c, 1-c$,

$$
x \text {-coordinate of centre } \quad=\frac{-c^{2} \alpha}{(1-\alpha)^{2}-c^{2}},
$$

which has the sign of $-\alpha$,

$$
\begin{array}{ll}
x \text {-semi-axis } & =\frac{ \pm c \alpha(1-\alpha)}{(1-\alpha)^{2}-c^{2}}, \\
y \text {-semi-axis } & =\frac{ \pm c \alpha}{\sqrt{\left\{(1-\alpha)^{2}-c^{2}\right\}},} \\
\text { semi-aperture of asymptotes } & =\tan ^{-1} \sqrt{\left\{1-\frac{c^{2}}{(1-\alpha)^{2}}\right\}},
\end{array}
$$

which for $\alpha=1 \pm c$ is $=0$ (parabola), but increases as $1-\alpha$ increases positively or negatively, becoming $=45^{\circ}$ for $\alpha= \pm \infty$ (the asymptotes being in this case the pair of lines $\left.y^{2}-x^{2}=0\right)$ :

$$
\begin{aligned}
& \alpha=+\infty, \text { coordinate of centre is }=0, \\
& \alpha=1+c, \quad, \quad, \quad=-\infty,
\end{aligned}
$$

so that $\alpha$ diminishing from $\infty$ to $1+c$, the coordinate of the centre moves constantly in the same direction from 0 to $-\infty$,

$$
\begin{aligned}
& \alpha=1-c, \text { coordinate of centre is }=-\infty, \\
& \alpha=0, \quad, \quad, \quad=0,
\end{aligned}
$$

the hyperbola being in this case the pair of lines $y^{2}=\left(1-c^{2}\right) x^{2}$.

$\alpha$ negative, the coordinate of centre becomes positive, viz. as $\alpha$ passes from $\alpha=0$ to $\alpha=-\sqrt{ }\left(1-c^{2}\right)$, the coordinate of centre passes from 0 to a maximum positive value $\frac{1}{2}\left\{1-\sqrt{ }\left(1-c^{2}\right)\right\}$, and then as a passes from $-\sqrt{ }\left(1-c^{2}\right)$ to $-\infty$, the coordinate of centre diminishes from $\frac{1}{2}\left\{1-\sqrt{ }\left(1-c^{2}\right)\right\}$ to 0 . It is to be remarked that $\alpha$ being negative, the lines $y^{2}-x^{2}=0$ are touched by the branch on the negative side of the origin, that is the branch not passing through the two points $x=1, y= \pm \sqrt{ }\left(1-c^{2}\right)$. 